\title{
EDITORIAL
}

\section{Antimicrobial Resistance in Nepal}

Antimicrobials are the most effective treatment for infectious diseases. Antimicrobial resistance (AMR) has increased and spread due to overuse and misuse of antimicrobials. Antimicrobial resistance especially multidrug resistance threatens the effective prevention and treatment of infections resulting in prolonged illness and increased mortality.

Studies revealed that major antimicrobial resistant pathogens in Nepal included Escherichia coli, Staphylococcus aureus, Klebsiella pneumoniae, Pseudomonas aeruginosa, Proteus spp., Salmonella spp., Shigella spp., Mycobacterium tuberculosis bacteria and Plasmodium falciparum, Leishmania donovani parasites.

In 2011, the South East Asia Region's health ministers adopted the Jaipur Declaration on Antimicrobial Resistance, which states that combating antimicrobial resistance must be a priority for national governments. The countries are introducing legislation and policies to govern the use of antimicrobial medicines; establishing laboratory-based networks for surveillance of antimicrobial resistance; ensuring rational use of antimicrobial medicines in all health care settings; and promoting community awareness about antimicrobial resistance.

Antimicrobial resistance surveillance was started in 1998 in Nepal with 9 laboratories and gradually included other laboratories selected on convenient criteria: size and place of the hospitals in the health system (regional, zonal, district hospitals), geographical access and coverage. Nepal has developed the National Antimicrobial Resistance Containment Action Plan, in continuation to its ongoing laboratory based AMR surveillance including 10 priority bacterial pathogens recorded and reported through existing 22 AMR sentinel sites.

One health approach involving coordination among numerous international sectors and actors, including human and veterinary medicine, agriculture, finance, environment, and well informed consumers is needed to prevent further emergence and spread of antimicrobial resistance. For preventing AMR, biosafety and biosecurity policies should be in place and AMR surveillance capacities should be strengthened and expanded in human and animal health sectors.

\section{Dr. Megha Raj Banjara}

Editor in chief

Tribhuvan University Journal of Microbiology (TUJM) 